\title{
Transatlantica
}

Revue d'études américaines. American Studies Journal

\section{Arshile Gorky ou le lyrisme inquiet}

Arshile Gorky - Hommage, du 4 avril au 4 juin 2007, Centre Georges

Pompidou et Centre Calouste-Gulbenkian.

\section{Mathilde Arrivé}

\section{(2) OpenEdition}

12 Journals

\section{Édition électronique}

URL : http://journals.openedition.org/transatlantica/2223

DOI : $10.4000 /$ transatlantica.2223

ISSN : 1765-2766

Éditeur

AFEA

\section{Référence électronique}

Mathilde Arrivé, "Arshile Gorky ou le lyrisme inquiet », Transatlantica [En ligne], 2 | 2007, mis en ligne le 03 février 2008, consulté le 29 avril 2021. URL : http://journals.openedition.org/transatlantica/2223 ;

DOI : https://doi.org/10.4000/transatlantica.2223

Ce document a été généré automatiquement le 29 avril 2021

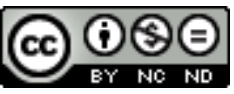

Transatlantica - Revue d'études américaines est mis à disposition selon les termes de la licence Creative Commons Attribution - Pas d'Utilisation Commerciale - Pas de Modification 4.0 International. 


\title{
Arshile Gorky ou le lyrisme inquiet
}

\author{
Arshile Gorky - Hommage, du 4 avril au 4 juin 2007, Centre Georges \\ Pompidou et Centre Calouste-Gulbenkian.
}

\section{Mathilde Arrivé}

1 Dans le cadre de l'année de l'Arménie en France sont exposées à Paris une

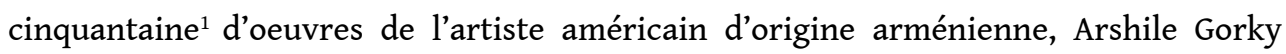
(1904-1948), né au bord du lac de Van et émigré aux Etats-Unis en 1920. Les huiles, pastels et dessins sont répartis dans deux espaces, selon deux grands temps : la période de formation (au centre Gulbenkian) et les oeuvres de la maturité de la "grande » période d'après 1943 (au centre Georges Pompidou). Cet hommage est donc une exposition en forme de parcours, permettant de suivre le cheminement artistique et individuel $\mathrm{du}$ peintre. A travers lui, c'est également toute la genèse de l'expressionnisme abstrait qui est retracée, de l'appropriation de la grammaire visuelle surréaliste et des leçons du cubisme vers leur reformulation américaine, au nom d'un idiome artistique national « original».

Hommage à un oublié du « triomphe $»^{2}$

2 L'inscription d'Arshile Gorky dans le mouvement expressionniste abstrait est problématique, car si à maints égards il en est l'un des initiateurs, il continue néanmoins de rester à l'orée de la grande école américaine. Dans la salle $1 \mathrm{du}$ Centre Pompidou, les œuvres de Gorky côtoient - de près mais à distance-les toiles monumentales d'un Pollock, d'un Rothko ou d'un Newman, artistes consacrés et emblématiques de l'école de New York, pour lesquels il n'est nul besoin d'hommage tant leur place dans l'histoire de l'art et dans l'institution muséale est tenue pour absolument légitime.

3 Si en 2007 Arshile Gorky reçoit les hommages du Musée national d'art moderne à Paris, il ne faut pas oublier qu'en 1945, lors de sa grande exposition à la galerie Julien Levy, Clément Greenberg lui réserva un accueil très défavorable dans un article de The Nation daté du 24 mars 1945, dans lequel il déclassait Gorky au rang de simple épigone des maîtres européens. Considéré comme un satellite des avant-gardes parisiennes, Arshile Gorky fut en effet disqualifié par l'école critique greenbergienne, alors si soucieuse de fabriquer une identité artistique américaine qui se définit en réaction à l'art cubiste et 
surréaliste, taxé d'intellectualisme stérile, de "féminité " précieuse, de conservatisme bourgeois ${ }^{3}$. Dans un contexte de guerre froide, les avocats de l'expressionnisme abstrait désirent en effet en finir avec la «colonisation » artistique de l'Amérique par l'Europe en promouvant un art que l'on dit volontiers libre, "liberal» et démocratique, entretenant dans le choix des adjectifs une confusion délibérée entre critères artistiques, valeurs morales, revendications identitaires et idéologiques. Ce qui se joue à travers la disqualification de Gorky, c'est donc le rejet symbolique de l'Europe et de sa «tutelle»-un rejet de principe. Car l'on veut croire qu'au sortir de la deuxième guerre mondiale,

l'art américain est devenu indépendant, autonome et il exprime avec authenticité la volonté, l'esprit et le caractère de ce pays. Il semble que stylistiquement, l'art américain ne soit plus un simple répertoire d'influences européennes, un simple amalgame d'«-ismes » importés de l'étranger, arrangés, recomposés et assimilés avec plus ou moins d'intelligence 4 .

Dans «le monde de l'art", la distribution des palmes et des blâmes consacre ou sanctionne impitoyablement. On peut alors comprendre cette double exposition déployée sur le mode de l'« hommage » comme une entreprise de réparation en forme d'honneurs différés, ou comme un geste de rappel, à la mémoire de cet artiste-passeur, maitre et ami du jeune De Kooning à la New School of Design et professeur de Rothko pour un temps à New York, qui a participé à l'introduction et à la refonte des idées et des formes européennes aux Etats-Unis et ainsi contribué au passage d'un provincialisme frileux et «moribond» à une peinture abstraite et expressionniste «triomphante ».

5 Provenant de la National Gallery of Art, du MoMA, du Metropolitan et du Whitney, les œuvres de Gorky sont rassemblées à Paris jusqu'au 4 juin 2007, sauvées de la dispersion pour l'occasion. C'est donc un nouvel espace de visibilité de l'œuvre du peintre américain qui est offert au public français, un moyen de découvrir ou de relire le travail d'un artiste méconnu aux Etats-Unis et presque inconnu en France. Si la filiation à l'expressionnisme abstrait n'est mentionnée que de manière allusive dans le communiqué de presse, c'est peut-être pour encourager à recevoir l'œuvre de Gorky en dehors d'une systématique référence au mouvement, en dehors de la problématique binaire de son inclusion/exclusion à l'école américaine. Car il s'agit en effet aujourd'hui de réinvestir ou de réactualiser l'œuvre de Gorky, de la soumettre à des relectures auxquelles de toute évidence elle se rend disponible.

La hantise des origines

6 Si l'on a rappelé que la critique américaine des années 1950 avait injustement disqualifié le peintre sur la base de ses influences européennes, il est en revanche incontestable que l'œuvre de Gorky est traversée d'inspirations diverses mais manifestes, sans que pour autant elle ne soit jamais simplement réductible à la somme de ses influences. C'est ainsi que Paul Cézanne, Fernand Léger, Roberto Matta ou George Braque, et plus tard, Joan Miró, Kandinsky, De Chirico et Picasso travaillent les productions de Gorky. On retrouve en effet dans les dessins au graphite Portrait of Vartoosh (1927) et The Philosophress (1932) un attachement à la figure et au trait académique, qui rappelle Picasso dans sa période classique, tandis que l'étude à l'encre de chine Nighttime, Enigma, Nostalgia (1932) et Painter and Model (1931) traduisent plutôt l'influence du Picasso cubiste. Qu'il s'agisse là d'un exercice d'emprunt ou de citation, Gorky s'engage dans un véritable dialogue, dans lequel sa voix propre émerge progressivement. Ce que Harold Bloom a pu nommer «l'anxiété de l'influence $»^{5}$ ne 
semble pas avoir inhibé Gorky, mais plutôt l'avoir stimulé dans sa quête d'un langage plastique singulier, où le travail des autres fonctionne plus en tremplin qu'en obstacle. Arshile Gorky. Painter and Model (The Creation Chamber), 1931. Lithograph on paper, $28.6 \times 25.1 \mathrm{~cm}$. Smithsonian Modern Art Museum, USA.

7 Même si les variations cubistes de Gorky lui valurent le surnom ingrat de « the Picasso of Washington Square $»^{6}$, il semble que se soit bien le surréalisme qui imprègne le plus son travail, ce qui occasionna le soutien enthousiaste d'André Breton. Garden in Sochi (1941), avec ses volutes biomorphiques aux couleurs franches, des volumes tout à la fois suggérés et niés, son atmosphère onirique, rappellent explicitement l'œuvre de Miró. ${ }^{7}$ Garden in Sochi, hymne nostalgique au jardin perdu de l'enfance, inspire à Gorky ces mots :

I like the heat the tenderness the edible the lusciousness the song (...)

The wheatfields, the plough, the apricots, the shape of apricots those flirts of the sun.

8 A cet imaginaire pastoral du jardin originel arménien va succéder un imaginaire plus explosif, plus sensuel mais aussi plus inquiet de la nature, lorsque le peintre rencontre le paysage américain dans Waterfalls (1943) et Landscape-Table (1945).

Arshile Gorky. Garden in Sochi, 1941. Oil on canvas, 64 x $74 \mathrm{~cm}$. The Museum of Modern Art, New York. (C) 2007 Estate of Arshile Gorky/Artists Rights Society (ARS), New York.

9 Avec Garden in Sochi, l'on comprend en tout cas pourquoi la tonalité lyrique, l'équilibre - presque statique - de la composition du tableau, l'harmonie des formes et l'infini travail de finition furent jugés par trop apolliniens pour les critiques d'art américain, en quête d'un art prosaïque, énergique et iconoclaste. La filiation européenne de Gorky ne pouvait en effet que le desservir dans une Amérique employée à « dé-définir » l'art ${ }^{8}$, pétrie du fantasme de la perpétuelle régénération ainsi que des vertus de la table rase dans l'entreprise définitoire de l'identité artistique américaine. A contre-courant de l'archétype romantique du génie inspiré et du talent spontané, Arshile Gorky est un homme de travail qui s'emploie tout au long de sa vie à étudier sans relâche l'œuvre de ses maîtres et de ses paires.

10 Contrairement aux apôtres de la « self-reliance » créatrice, Gorky n'est pas dans une logique de rupture mais revendique plutôt l'importance de la continuité et de la mémoire. Il demeure en effet toujours ancré dans les origines, autant au niveau des sujets que de leur traitement stylistique. Qu'il s'agisse de la mémoire douloureuse du génocide arménien ou de la mort de sa mère (comme dans Agony de 1947 ou The Vale of the Armenians de 1944), ou de la mémoire nostalgique d'une Arménie rurale, solaire et généreuse (comme dans Cornfield of Health de 1941), le temps et le lieu des origines fonctionnent comme matrices à son travail. Habité par le passé et les héritages qu'il parvient à investir d'énergies neuves, Arshile Gorky nous offre du même coup des propositions visuelles tout à la fois étranges et familières.

Le pari de la synthèse et le travail de passage

11 Si l'on refuse à Gorky l'aura romantique du "self-made artist », on s'accorde néanmoins à reconnaitre sa fonction de charnière entre les cultures moyen-orientales et occidentales et de pivot entre l'art européen d'avant guerre et l'art américain d'après guerre. Considéré comme « le dernier surréaliste et le premier expressionniste abstrait », on le retient avant tout comme un passeur. Héritier de l'écriture automatique surréaliste, Gorky contribue à réhabiliter aux Etats-Unis le potentiel créatif des forces de l'inconscient, de l'instinct, de l'imagination et du corps. Par ailleurs, il participe 
aussi à une reformulation des absolus formels que sont le volume, la perspective, la figuration académique et le réalisme de la couleur et de la forme, qui prévalent largement dans l'art provincialiste américain d'avant guerre.

Arshile Gorky. Cornfield of Health, 1944. Oil on canvas, 86.5 x $111.5 \mathrm{~cm}$. Private collection.

12 Avec les couleurs primaires de Cornfield of Health, le rythme des pleins et des vides, les délinéations fébriles du trait noir, la fermeté vigoureuse de certains aplats, la transparence des lavis, Arshile Gorky offre une proposition visuelle syncrétique (qui d'ailleurs rappelle certaines toiles de De Kooning - à moins, bien sûr, que ce ne soit le contraire). Si le titre du tableau est partiellement référentiel ${ }^{9}$, Arshile Gorky s'abstrait dans son traitement du thème de la stricte dénotation en produisant ce qui s'apparente plus à un champ coloré ( color field») qu'à un champ de maïs (" cornfield»). La couleur déborde des traits qui ne la circonscrivent plus, occasionnant ainsi une série de déplacements qui confèrent du dynamisme à la composition. Dans Cornfield of Health, et à travers l'ambiguïté et la polysémie du terme de "champ", on perçoit déjà la tension-structurelle et fondatrice pour l'expressionnisme abstrait-entre une dimension corporelle, organique, sensuelle, parfois érotique, et une dimension cosmique, spirituelle, voire métaphysique. Les deux aspects convergent finalement dans une surface explosive, sur laquelle les couleurs résonnent et irradient, dans une matière picturale tantôt très texturée, tantôt très diluée.

D'autre part, si Gorky travaille principalement sur des toiles de moyenne dimension (ce qui le rattache à la "peinture de chevalet» si parisienne, si décriée), il a progressivement recours à des formats plus vastes, par lesquels le processus et le geste de peindre n'essaient plus de se dissimuler mais s'affirment comme ingrédients à part entière du tableau, et ce, dans une assertion de plus en plus marquée (et très greenbergienne) de la planéité bidimensionnelle de la toile. L'illusion perspective est abandonnée, l'espace pictural se déstructure, la composition se déhiérarchise, le regard s'égare, les traits se disloquent et s'épaississent, la géométrie cubiste s'arrondit et se rompt, au profit de tableaux sans centre, que l'on appellera bientôt « all-over ». Arshile Gorky, Waterfall. Oil on canvas, 1537 × $1130 \mathrm{~mm}$. Tate Gallery.

Dans Waterfall (1943), toute la surface de la toile est saturée de matière colorée, le geste est vigoureux, les contours disparaissent, les premières coulures de peintures apparaissent (ancêtres ou avatars des drippings de Pollock), convergeant vers la suggestion d'une énergie, par laquelle le tableau se donne comme champ de force rythmé, ponctué de zones d'intensités. C'est désormais la couleur, affranchie de toute délinéation, qui structure le champ pictural, si bien qu'avec Waterfall on se place déjà à la frontière de l'«Action painting » et de la peinture "Color field», dans une œuvre qui, en 1943 , reste pionnière.

Le spectre de la figure

15 Pour définir le travail de Gorky, l'on a parlé d'«abstraction figurative». Si cette étiquette définitoire en forme d'oxymore pose plus de problèmes qu'elle n'en résout, elle rend néanmoins compte de la tension dialectique entre figuration et abstraction chez Gorky.

Arshile Gorky. Landscape-Table, 1945. Oil on canvas, 92 x 121 cm. Musée national d'art moderne, Paris. (C) Collection Centre Pompidou, Paris.

16 Dans cette Landscape-Table (1945), l'on distingue certes une table, mais la dite table fonctionne plus en emblème du tableau, envisagé comme vaste réceptacle d'affect, de 
formes et de couleurs. La table représentée dans le tableau serait donc l'indice d'un commentaire réflexif sur le statut du tableau comme support (tabulaire, en l'occurrence). Et c'est ainsi que s'opère le glissement, cher à l'expressionnisme abstrait, de la question $\mathrm{du}$ "que peindre " (pour quel sujet) à celle du «comment peindre $»^{10}$ (par quel processus créateur) dans un travail qui se fait de moins en moins décoratif et de plus en plus expressif. Dans le même temps, de manière solidaire, on glisse de l'accent mis sur le signifié (" table ») à l'accent mis sur le strict signifiant coloré. De la figure, on passe au figural. ${ }^{11}$

Arshile Gorky. Aesthetics, c. 1946. Pencil and colour crayon on paper, 48.3 x $62.2 \mathrm{~cm}$. Calouste Gulbenkian Foundation, Lisbon.

17 En s'acheminant vers une esthétique de l'expression et des effets, Gorky entretient, on l'a dit, une relation ambiguë à la figure, qui toujours persiste alors même qu'elle tend à s'effacer ou se fondre dans la couleur. Dans Aesthetics (1946), il y a non-coïncidence entre le dessin et les zones de couleurs, qui se superposent et se décalent, travaillant ensemble mais séparément, de manière dissonante mais dynamique ${ }^{12}$. Et si le peintre fait persister la figure - ou ne veut pas la faire disparaître tout à fait - c'est peut-être aussi pour mieux jouer de ses distorsions. Car ce qui intéresse Gorky, ce n'est pas l'abstraction per se, mais la représentation, au sein de ses toiles, du passage à l'abstraction, où l'œuvre s'offre comme laboratoire des tensions qui la fondent. En ce sens, l'œuvre de Gorky cristallise les grandes «étapes » de l'art moderne-elle dramatise et rejoue sa difficulté à défaire la figure. Si l'abstraction fut accueillie par ses défenseurs américains comme une libération et l'ultime conquête de l'art moderne, Arshile Gorky énonce quant à lui la quasi-disparition de la figure sur le mode mélancolique d'une perte augurant toujours de possibles retours.

D'autre part, dans Aesthetics, le dessin d'un visage stylisé (et clownesque) semble apparaître de manière spectrale comme en filigrane sous la couleur, alors même qu'il a bien été ajouté au crayon sur le fond au pastel. A travers ce dessin, aux allures de palimpseste, on a comme un feuilletage de la surface, la suggestion d'un contenu latent, l'indice d'un sous-texte ou d'un inter-texte figuratif. Finalement, c'est bien la fragilité du trait associée à la nébuleuse colorée qui confèrent au travail de Gorky une grande finesse et le distinguent ainsi des toiles plus chargées d'autres artistes expressionnistes abstraits.

Respirations, essoufflement et « agonies »

19 Certaines œuvres d'Arshile Gorky sont sources de fascination. En témoignent les dessins au graphite et pastel, Study for the Calendars, Study for Summation de 1946 ou encore le dessin au titre merveilleux de « Dance of the Eyelids». Ces dessins se donnent comme des « espaces à la fois sensuels et chiffrés » où se déploie une étrange écriture, une pulsation presque mélodique. Ces dessins rappellent, dans leur composition absolument centripète, l'aléatoire et la sophistication de l'écriture automatique surréaliste. Ils témoignent en outre d'un vrai souci du détail-des détails qui adviennent comme autant de petits événements, voire d'accidents discontinus ou de minuscules déflagrations.

Arshile Gorky. Study for Summation, 1946. Pencil and crayon on paper, 46.7 x $61 \mathrm{~cm}$. The Museum of Modern Art, New York. (C) 2007 Estate of Arshile Gorky/Artists Rights Society (ARS), New York.

Lorsque l'on regarde ces dessins, on imagine les torsions du poignet de l'artiste éraflant le papier de petits coups de crayon, au point que l'on pourrait parler ici d'« Action 
Drawing ", en écho à l'« Action Painting " cher à Harold Rosenberg. On se hasardera également à lire dans cette "danse des paupières" un prélude aux vastes chorégraphies de Pollock qui danse, cette fois de tout son corps, sur sa toile et autour d'elle, prenant les filets de peinture liquide pour partenaire. Il y a dans ces ballets en miniature (ces pantomimes?) semés de légères ponctuations colorées, quelque chose qui rappelle aussi une nuée de petits insectes, bruissante et drolatique.

Chez Gorky, le papier est effleuré, parfois griffé, mais aussi biffé, puisque là encore quelque chose semble s'annuler en se traçant. Il y a en effet quelque chose de très fragile, de précaire et d'apparemment éphémère dans cette transcription de frémissements d'énergie fugaces, incertains. Ces dessins, qui donnent une impression d'inachevé, tirent précisément toute leur force de ne point avoir êté trop travaillés, d'être restés comme suspendus.

Ce qui frappe enfin, c'est la tonalité grise qui tranche avec la luminosité et la fraîcheur des couleurs chaudes des autres tableaux. On l'a dit, pas d'allégeance stylistique stricte chez Gorky, pas de dogmatisme. Dans la série des tableaux gris, on retrouve certes les études au graphite, mais également des huiles (Days, etc de 1947 et Diary of a Seducer de 1945).

Arshile Gorky. Diary of a Seducer, 1945. Oil on canvas, 126.7 x $157.5 \mathrm{~cm}$. The Museum of Modern Art, New York.@ 2007 Estate of Arshile Gorky/Artists Rights Society (ARS), New York.

Le journal d'un séducteur, au titre dramatique et narratif, offre une palette diluée de gris et de noirs, un trait plus dur - plus nonchalant aussi - un univers clos et caverneux, un espace saturé, une composition plus «centrée», régie par un mouvement descendant. S'il est toujours question d'énergie, il semble que ce soit une énergie quelque peu entropique, exprimant une angoisse, une fatigue - l'essoufflement d'une volupté mélancolique et désenchantée. Pourtant dans cette toile, ce sont précisément les zones sombres qui créent la lumière, vers la suggestion d'un scénario presque existentiel. Car Arshile Gorky incarne, s'il en est, "l'homme existentiel» par excellence : son destin est celui d'un grand amoureux et d'un affabulateur acharné qui se suicida par pendaison en 1948, alors qu'il est frappé par le cancer et qu'il ne parvient pas à se relever d'un mariage en déroute, d'un incendie qui détruit une grande partie de son travail et d'un accident qui le laisse dans l'impossibilité de peindre.

Il serait bien sûr vain de vouloir trouver dans la peinture d'Arshile Gorky les indices d'une biographie tourmentée. Cependant, comment ne pas terminer ce compte rendu par l'évocation d'un de ses derniers opus, Agony (1947), dans lequel la douleur s'énonce cette fois sur un mode flamboyant, incandescent. Agonie est un tableau janus qui à la fois se retourne une dernière fois vers la douleur du génocide arménien, mais qui laisse également présager la mort prochaine du peintre.

Arshile Gorky. Agony, 1947. Oil on canvas, 101.6 x $128.3 \mathrm{~cm}$. The Museum of Modern Art, New York. (c) 2007 Estate of Arshile Gorky/Artists Rights Society (ARS), New York.

25 Ce tableau fonctionne en creuset (Agony s'apparente à un grand feu) dans le rassemblement des ingrédients disparates que le peintre opère, dans une recherche jamais stabilisée de la synthèse - des styles, des formes, des couleurs et des affects. 


\section{NOTES}

1. Une vingtaine de toiles sont présentées au Centre Pompidou, auxquelles s'ajoutent une trentaine de dessins au Centre Gulbenkian.

2. J'emploie ici le terme de « triomphe » en référence - ironique - au titre de l'ouvrage d'Irving Sandler, Le triomphe de l'art américain (Paris : éditions Carré, 1990), qui, dans le sillage de Clément Greenberg, voit dans l'expressionnisme abstrait le point culminant de l'hégémonie artistique américaine après la deuxième guerre mondiale.

3. Pour Clément Greenberg, les jeunes artistes américains doivent éviter l'académisme, le néo-romantisme et le surréalisme dans lequel il ne voit que « de nouvelles anecdotes à illustrer qui favorisent la réhabilitation de l'art académique sous un déguisement littéraire ", The Nation $69: 8$ (août 1944), 219-220. Au sujet de la théorie « moderniste » de Clément Greenberg, voir Art and Culture (Boston : Beacon press, 1961) qui rassemble une quinzaine d'essais écris entre 1948 et 1958 par le critique et théoricien.

4. Frederic Taubes, articles « Painting », Encyclopaedia Britannica, «Book of the Year $1946 », 573$.

5. Cf. Harold Bloom, The Anxiety of Influence : a Theory of Poetry (New York : O.U.P, 1973). 6. Hayden Herrera, Arshile Gorky, His Life and Work (New York : Farrar, Straus \& Giroux, 2003) «The Picasso of Washington Square » est également le titre d'une recension de ce même ouvrage par Andrew Solomon.

7. De même que dans sa vie, Gorky fut un grand fabulateur, bricolant une identité composite à partir de différents récits souvent contradictoires, sa peinture est comme tapissée par les images des autres et la relecture singulière qu'il en donne : sur les toiles comme chez l'homme, les évolutions ne sont pas linéaires, les influences ne sont pas exclusives, le style n'est jamais systématique.

8. Cf. Harold Rosenberg. La dé-définition de l'art (Nîmes : éditions Jacqueline Chambon, «Rayon art », 1992). Voir également du même auteur, La tradition du Nouveau (Paris : éditions de Minuit, 1962).

9. « Cornfield of Health » est traduit en français par « Champ de maïs vivifiant ».

10. Robert Motherwell, interviewé par Max Kozloff, dans Artforum $4: 1$ (septembre 1965), 37.

11. Jean François Lyotard, Discours, Figure (Paris : édition Klincksieck, (4e tirage), 1985), 13

12. Dans ce tableau en forme de traité de l'art pictural, Arshile Gorky semble nous dire, à travers le choix de son titre, que l'« esthétique » résulte bien de l'affrontement fécond et toujours reconduit entre ces deux forces « traditionnelles » que sont le trait et la couleur. L'opposition entre trait et couleur, dont Arshile Gorky semble offrir un dépassement, fait problème depuis Aristote. 
INDEX

Thèmes : Trans'Arts

\section{AUTEUR}

MATHILDE ARRIVÉ

Université Bordeaux 3 